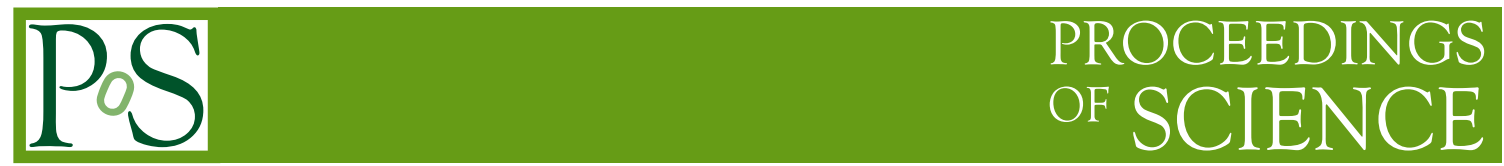

\title{
Early Electroweak Signals at the LHC
}

\author{
Maarten Boonekamp ${ }^{* \dagger}$ \\ CEA, IRFU/SPP \\ E-mail: maarten.boonekamp@cea.fr
}

I review the current uncertainties on electroweak boson production at the LHC, and how the foreseen measurements will help to improve the situation, event with modest integrated luminosities.

XXth Hadron Collider Physics Symposium

November 16 - 20, 2009

Evian, France

${ }^{*}$ Speaker.
'On behalf of the ATLAS and CMS Collaborations. 


\section{Introduction}

The aim of this contribution is a review of electroweak boson production at the LHC, the uncertainties that remain, and how the LHC data will extend our knowledge in this field.

The study of Drell-Yan interaction in its general acception (lepton pair production from virtual photon, $\mathrm{W}$ and $\mathrm{Z}$ exchange) has made significant progress in recent years. The theoretical description of hard the process has been brought to next-to-next-to-leading order (NNLO) [1], and our knowledge of the proton parton density functions (PDFs) is improving thanks to the ongoing combination of the complete HERA data set [2], and to the improving precision of the Tevatron data [3]. It is claimed that uncertainty on the overall $\mathrm{W}$ and $\mathrm{Z}$ production gets $O(1 \%)$ from the hard process calculation, and $O(5 \%)$ from the parton density functions, restricting the use of these processes to sources of experimental calibration (the detector response to leptons can be extracted from the observed $\mathrm{Z}$ linesdhape; the production rate contributes to the luminosity determination). We question these statements below. We briefly review the assumptions hidden behind the claims, and show the impact of the LHC measurements on various examples. In many cases, measurements are interesting already with $O(50) \mathrm{pb}^{-1}$ of data.

\section{Current uncertainties on electroweak boson production}

The HERA Experiments are currently finalizing the combination of their complete structure function data sets, with significant gain in precision on the PDFs. As illustrated in Figure 1, the resulting uncertainty bands are often twice as small as those provided by recent global analyses [3,4], indeed leading to uncertainties of at most 5\% on Drell-Yan production at the LHC. It should however be noted that what is measured at Hera is a particular combination of parton densities, namely $F_{2} \sim 4(u+c)+(d+s)$. Up to some scarce heavy flavour data $\left(F_{2}^{c c, b b}\right)$ and neutrino scattering data [6], the contributions of the second generation partons are not well constrained, and assumptions are made: usually, one assumes proportionality of the $s$ density to the sum $\bar{u}+\bar{d}$.

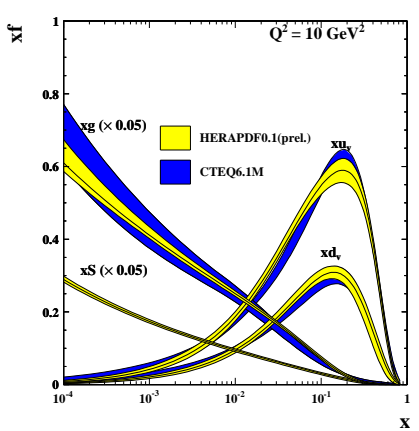

(a)

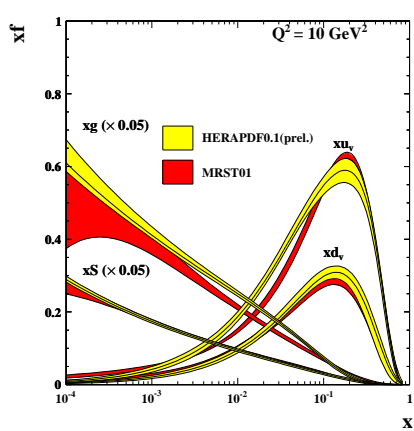

(b)

Figure 1: Current proton parton density measurements. HERA compared to CTEQ6M (left), and to MSTW01 (right).

This is well visible in Figure 2, showing the strange density at $x, Q^{2}$ relevant for $\mathrm{W}$ and $\mathrm{Z}$ production at the LHC obtained when releasing the proportionality assumption. The uncertainty on this density 
is $O(25 \%)$ everywhere. This is particularly important at the LHC which, being a $p p$ machine, does not benefit from valence-valence interactions, so that the process $s c \rightarrow W$ is responsible for about $25 \%$ of the overall $\mathrm{W}$ production, for example. Considering the remaining uncertainties on $u, d$ and $c$ densities, $5 \%$ PDF uncertainty on $\mathrm{W}$ prodution seems a lower bound.

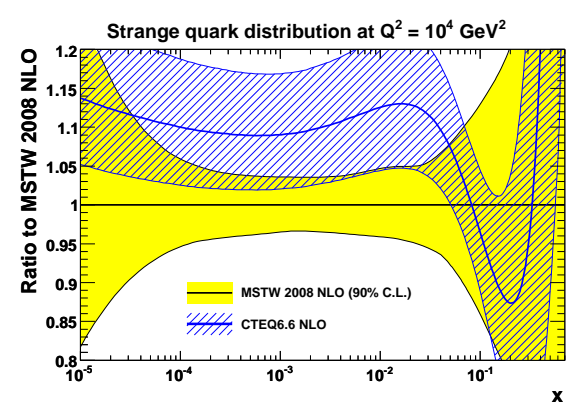

Figure 2: Strange parton density: MSTW08 compared to CTEQ6.6.

The treatment of heavy quarks in the PDF evolution is also theoretically sensitive. In the study leading to the CTEQ6.5 PDF sets [5], the implementation of the so-called general mass variable flavour number scheme (GM-VFNS) lead to an increase of the $\mathrm{W}$ and $\mathrm{Z}$ cross sections by about $8 \%$. While this number reflects an improvement rather than an uncertainty, one wonders if further improvements can still induce such strong variations.

Finally, the uncertainty on the hard process, evaluated to $O(1 \%)$ in [1], can be questioned too. This result was obtained by assuming, as usually done, that the factorization and renormalization scales are identical, and varying these scales within a factor two. Reproducing this exercise and letting the two scales vary independently within the same range, however, produces cross section variations of about 5\%, as shown by studies performed within the ATLAS Collaboration.

\section{Total cross section measurements}

It remains thus important to measure these well-known processes. As we wil see, the expected rates are such that the statistical uncertainty will soon become small, and the analyses will be almost entirely focus on the reduction of the systematic uncertainties. In this context, we argue that the measurement of the total cross sections is of limited interest in a first stage, while the differential cross sections provide more insights; including constraints from the differential measurement, the total cross sections become more precise.

Our two following examples are taken from the ATLAS "CSC book" [7]. The first one, $\mathrm{Z} \rightarrow \mu \mu$, is a rather clean process, and can be selected with high efficiency and low backgrounds. The selection requires two well identified, high- $p_{T}$, well isolated muons of opposite chage $\left(p_{T}>25 \mathrm{GeV}\right.$, $|\eta|<2.5$ ). Figure 3 (left) shows the invariant mass distribution ahead of the isolation cuts; the background is negligible after this cut. The process itself can be used to measure the muon selection efficiency, through the so-called "tag and probe" method, relying on the tight selection of one lepton, and the loose selection of the second one to determine the efficiency of the released cuts. 
The result of this procedure is illustrated in the right pane of Figure 3, where good consistency between the true (Monte Carlo based) and measured efficiency is observed.

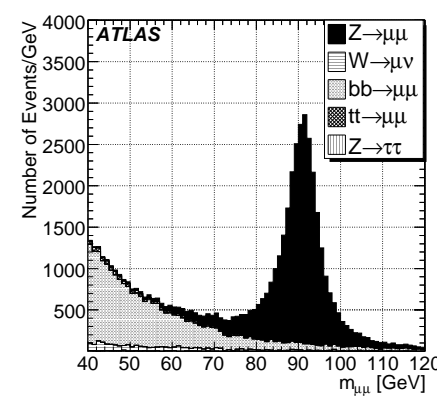

(a)

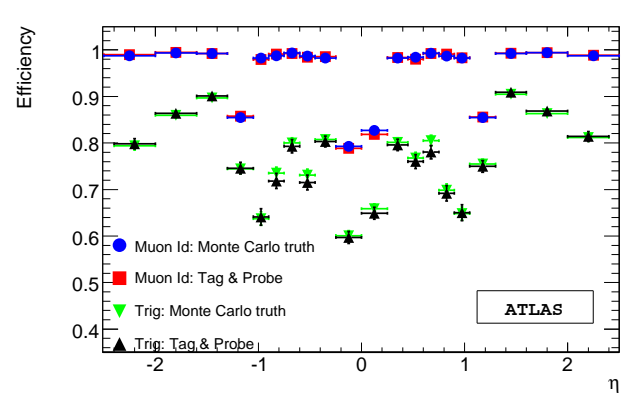

(b)

Figure 3: The $\mathrm{Z} \rightarrow \mu \mu$ lineshape (a), and the muon trigger and offline efficiencies (b) .

After these selections, and assuming $100 \mathrm{pb}^{-1}$ at $7 \mathrm{TeV}$, about 26000 events are expected, with a statistical uncertainty of about $0.8 \%$. Backgrounds are small; the main systematic uncertainties come from the selection efficiency, $\sim 2 \%$, and from the theoretical description of the process, $\sim 2.3 \%$, which induces uncertainties on the selection acceptance. The efficiency uncertainty will reduce statistically with more data, but the acceptance uncertainty can not shrink without further input.

Our second example is the $\mathrm{W} \rightarrow e v$ process, illustrated in Figure 4. The selection requires one identified electron of high transverse momentum, significant missing transverse energy from the decay neutrino, and high transverse mass of the electron-neutrino system ( $p_{T}>25 \mathrm{GeV},|\eta|<2.4$, $E_{T}^{\text {miss }}>25 \mathrm{GeV}, M_{T}>40 \mathrm{GeV}$ ). The expected transverse mass distribution is illustrated in Figure 4 (left). In this case, where only one reconstructed lepton is required, the backgrounds are higher, and difficult to predict as they mainly come from the tails of jet production and affected by strong interaction and fragmentation uncertainties. The background level needs to be determined from the data themselves. One possibility is to extract from a signature free of $\mathrm{W}$ signal, and sensitive the same background; such a sample can be extracted using photon triggers and loose selections. As shown in Figure 4 (right), the jet background under the $\mathrm{W}$ and the sample selected as above have a very similar shape, allowing to estimate the background.

With the same assumptions as above, about 230000 events are expected, with an uncertainty of $0.2 \%$. Accounting for the background determination in situ, the acceptance uncertainty is again dominant and irreducible without further input.

\section{Differential cross sections}

The acceptance uncertainties affecting the total cross section measurements above are essentially induced by PDF uncertainties. Uncertainties in the $x$-dependence of the parton densities affect the $\mathrm{W}$ and $\mathrm{Z}$ rapidity distributions, translating into the quoted acceptance uncertainties. That is, total cross section measurements are hindered by the very effect they should constrain. This indicates 


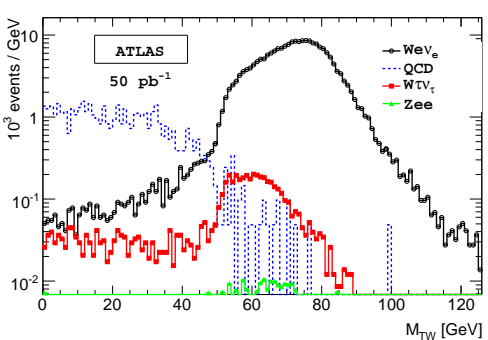

(a)

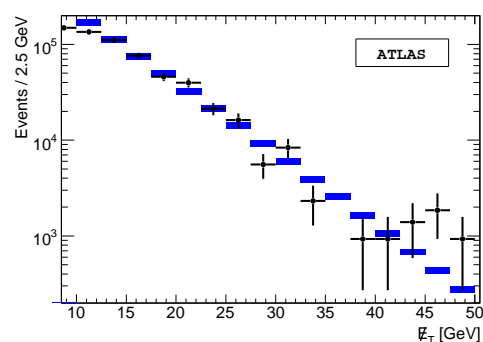

(b)

Figure 4: The $\mathrm{W} \rightarrow e \mu$ transverse mass distribution (a). Estimation of the jet background under the $\mathrm{W}$ (b) .

the need to measure the distributions precisely, before feeding them back to the total cross section measurement. Other long term applications like the $\mathrm{W}$ mass measurement also benefit from these measurements [8].

The examples below are taken from the CMS collaboration [9]. One interesting and robust measurement to be performed is the charge asymmetry in leptonic $\mathrm{W}$ decays, defined as follows:

$$
A(\eta)=\frac{N\left(\mu^{+}\right)-N\left(\mu^{-}\right)}{N\left(\mu^{+}\right)+N\left(\mu^{-}\right)}
$$

and is sensitive to the valence quark distributions, $u_{v}-d_{v}$. Here, most experimental uncertainties, in particular the lepton selection efficiency, cancel in the ratio. The muon angular resolution is negligible. The main uncertainty comes from the background which is charge symmetric, contrarily to the signal, and dilutes the asymmetry. This background can be subtracted precisely, as in the previous example, but leaves the dominant systematic uncertainty due to the cancelation of the other effects.

The expected measurement result, assuming $100 \mathrm{pb}^{-1}$ at $10 \mathrm{TeV}$, is illustrated in Figure 5 (left). The expected precision is compatible with the PDF uncertainties. Note that the PDF set used in this study assumes fixed strangeness as discussed above, hence probably underestimates the true uncertainty. Since the systematic uncertainties are, here again, determined from auxiliary measurement, higher luminosity will bring higher precision.

Finally, we mention the $\mathrm{Z}$ boson rapidity distribution measurement (Figure 5, centre and right plots). In the electron channel, the possibility to measure electrons in the forward calorimeters makes this measurement interesting. While almost flat in the central rapidity region, the distribution becomes more steep and uncertain at high rapidity. Measurements in this region constrain the PDF uncertainties in a way complementary to the charge asymmetry discussed above. As illustrated by the uncertainty breakdown, the theoretical uncertainties are here small compared to the experimental ones, in contrast to the previous section, illustrating once more the relevance of the differential measurements.

To end this section, let us remind that the various center-of-mass energy runs currently planned by the $\operatorname{LHC}(7,10,14 \mathrm{TeV})$ are an opportunity in this context, as each configuration probes a given parton $x$ range, so that measurements at different $\sqrt{s}$ will bring complementary information. 


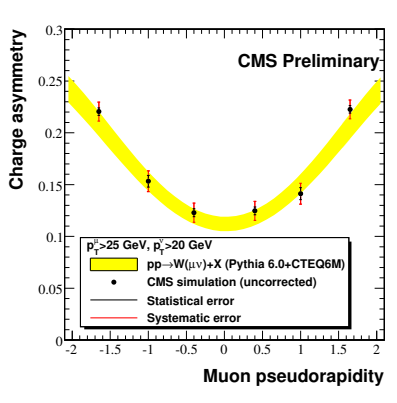

(a)

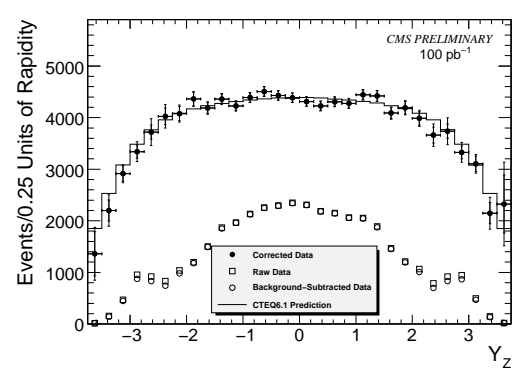

(b)

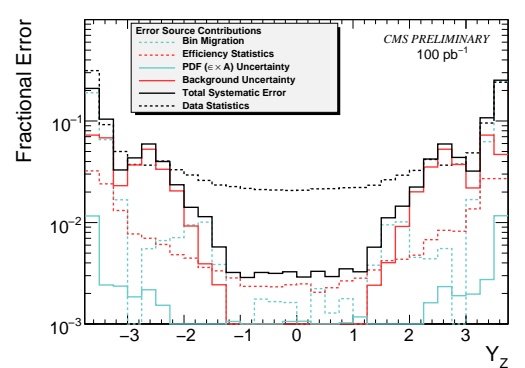

(c)

Figure 5: Measured asymmetry in the $W \rightarrow \mu v$ channel (a). Measured rapidity distribution in the $\mathrm{Z} \rightarrow e e$ channel (b), and the contributions to the total uncertainty (c).

\section{Conclusions}

To summarize the arguments of this contribution: the uncertainties on $\mathrm{W}$ and $\mathrm{Z}$ production at the LHC are larger than commonly quoted, and these processes deserve to studied with the greatest possible precision. Differential cross section measurements are essential to constrain the uncertainties affecting the distributions, notably the PDF shapes. Once this is done, total cross section measurements benefit from reduced acceptance uncertainties and become relevant to constrain the process normalization. The envisaged centre of mass energies are a real opportunity that extends the physics reach of the LHC experiments.

I am grateful for the help of my ATLAS and CMS colleagues in the preparation of this contribution, and to the conference organizers for their invitation.

\section{References}

[1] Kirill Melnikov, Frank Petriello; Phys.Rev.D74:114017,2006; hep-ph/0609070.

[2] HERA Combined Structure Functions Working Group (C. Gwenlan for the collaboration); Nucl.Phys.Proc.Suppl.191:5-15,2009; axXiv:0902.1807.

[3] A.D. Martin, W.J. Stirling, R.S. Thorne, G. Watt; Eur.Phys.J.C63:189-285,2009; arXiv:0901.0002.

[4] Pavel M. Nadolsky, Hung-Liang Lai, Qing-Hong Cao, Joey Huston, Jon Pumplin, Daniel Stump, Wu-Ki Tung, C.-P. Yuan; Phys.Rev.D78:013004,2008; arXiv:0802.0007.

[5] W.K. Tung, H.L. Lai, A. Belyaev, J. Pumplin, D. Stump, C.-P. Yuan; JHEP 0702:053,2007; hep-ph/0611254.

[6] NuTeV Collaboration; Phys.Rev.D74:012008,2006; hep-ex/0509010.

[7] ATLAS Collaboration, CERN-OPEN-2008-020.

[8] ATLAS Collaboration (N. Besson et al.); Eur.Phys.J.C57:627-651,2008; arXiv:0805.2093.

[9] CMS Collaboration, CMS-PAS-EWK-09-001, 003, 004, 005, 006. 